\title{
A Distributed Command Governor Strategy for the Operational Control of Drinking Water Networks
}

\author{
Francesco Tedesco, Carlos Ocampo-Martinez, Alessandro Casavola and Vicenç Puig
}

\begin{abstract}
This paper proposes the application of a distributed command governor (DCG) strategy for the operational control of drinking water networks (DWN). This approach is very suitable to this kind of management problems given the large-scale and complex nature of DWNs, the relevant effect of persistent disturbances (water demands) over the network evolutions and their marginal stability feature. The performance improvement offered by DCG is compared with the consideration of two non-centralized model predictive control (MPC) approaches already proposed for the same management purposes and within the same context. The paper also discusses the effectiveness of all strategies and highlights the advantages of each approach. The Barcelona DWN is considered as the case study for the assessment analysis.
\end{abstract}

\section{INTRODUCTION}

Drinking Water Networks (DWNs) are in charge of bringing water from production plants to consumers. From a dynamical systems point of view, DWNs are large-scale multiinput/multi-output interconnected flow systems that must be made reliable and resilient to continuously varying customer behaviours, of both deterministic and probabilistic nature, and various operational constraints. Several approaches have been reported in the literature that address operational control problems for DWNs; see, e.g., [1], [2], [3], [4], [5], [6], [7] for relevant contributions. Among many others, decision policies inspired by the model predictive control (MPC) framework [8] are considered to be quite suitable to face the operation of DWNs due to their flexibility to manage constraints and minimize multi-objective cost indexes found in the optimization of these complex systems, see [4].

In this paper, the Command Governor (CG) approach is considered for the operational control of DWNs [9] and aimed at performing comparisons with strategies based on MPC control ideas. The CG approach has several similarities with MPC control, the most relevant being the fact that its action is computed by solving on-line a constrained convex optimization problem based on future system predictions. However, the two approaches have also remarkable differences that make the CG approach computationally less

\footnotetext{
F. Tedesco and A. Cassavola are with University of Calabria, DIMES, 87036 Rende (CS), Italy. \{tedesco, casavola\}@dimes.unical.it

C. Ocampo-Martinez and V. Puig are with Automatic Control Department, Universitat Politècnica de Catalunya (UPC), Institut de Robòtica i Informàtica Industrial (CSIC-UPC), Llorens i Artigas 4-6, 08028 Barcelona, Spain. \{cocampo, vpuig\}@iri.upc.edu

This work has been partially supported by the European Commission, the European Social Fund and the Calabria Region. The authors are solely responsible for the content of this paper and the European Commission and Calabria Region disclaim any responsibility for the use that may be made of the information contained therein.
}

demanding than MPC for the same problem at the price of reduced control performance. A quantification of this performance gap on a relevant DWN case study is one of the main goals of this paper.

In [10], a centralized CG solution for the operational control of DWNs has been proposed and compared with a centralized MPC scheme. Centralized solutions require a global dynamical model of the system for control design. Moreover, all measurements must be collected in one location to estimate all states and compute all control actions. However, when considering large-scale systems such as DWNs, these assumptions usually fail to hold, either because gathering all measurements in one location is not feasible or because a centralized high-performance computing unit is not available. Centralized schemes have also poor scalability properties and require the complete retuning of the controller also for small plant changes. Thus, the cost of setting up and maintaining a monolithic centralized controller could be prohibitive. A way of circumventing or mitigating these issues is to look into decentralized or distributed techniques, where network local controllers are in charge of the control of part of the entire system. Several works have been recently published in this area; see, e.g., [11].

In this paper, a distributed version of the CG (from now on called DCG) approach is described and used to face the operation of a DWN. In this case, the DWN is supposed to be partitioned into several subsystems, each of which is locally supervised by a single agent [12]. Early works on DCG strategies relied on a non-cooperative game theoretical approach and several strategies have been singled out, both sequential [13] (where, according to a prefixed order, only one agent at each sampling time is allowed to update its control action while all others keep applying their previous applied commands), and parallel [14] (where, on the contrary, all agents update their control actions simultaneously at each sampling time). Improved methods have recently been presented in [15] and [16]. Following the same lines of these works, in this paper a DCG strategy is proposed where agents are grouped into turns and where the entire decision process ends up within few sampling steps. There also will be shown that this method is also effective in the case of dynamic coupling among subsystems.

The remainder of the paper is organized as follows. The problem of the operational control of DWNs is stated in Section II. The proposed DCG approach is introduced in Section III. In Section IV, the results achieved for the Barcelona DWN case study are presented along with comparisons with other non-centralized MPC approaches. Finally, some 
conclusions and future research lines are presented in Section V.

\section{DWN Operational Control Problem STATEMENT}

\section{A. Control-oriented Modelling}

The DWN modelling approach proposed in [17] is briefly recalled. A general DWN can be represented by a directed graph $G(\mathcal{V}, \mathcal{E})$, where a set of elements, i.e., $n_{s}$ sources, $n_{x}$ storage elements, $n_{q}$ intersection nodes and $n_{d}$ sinks represent the vertices $v \in \mathcal{V}$ that are connected by direct links $a \in \mathcal{E}$. For the network operation, water is transported along the links by using $n_{u}$ flow actuators (i.e., pipes and valves), stored into reservoirs or tanks, taken from given sources and feeding specific destination locations. The network is subject to several capacity and operational constraints and affected by disturbances, such as measured stochastic flows to sinks driven by customer water demands.

Collecting water volumes contained in the storage elements into the state vector $\mathbf{x}(k) \in \mathbb{R}^{n_{x}}$, flows through actuators into the manipulated input vector $\mathbf{u}(k) \in \mathbb{R}^{n_{u}}$, and considering the demand flows as additive measurable disturbances collected into $\mathbf{d}(k) \in \mathbb{R}^{n_{d}}$, the DWN controloriented model may be described by the following set of linear (or linearised) discrete-time difference-algebraic equations (DAE) for all time instant $k \in \mathbb{N}$ :

$$
\begin{aligned}
\mathbf{x}(k+1) & =\mathbf{A} \mathbf{x}(k)+\mathbf{B} \mathbf{u}(k)+\mathbf{B}_{d} \mathbf{d}(k), \\
\mathbf{0} & =\mathbf{E}_{u} \mathbf{u}(k)+\mathbf{E}_{d} \mathbf{d}(k),
\end{aligned}
$$

where (1a) describes the dynamics of storage tanks whereas the algebraic equations (1b) describe the network static relations (i.e., mass balance at junction nodes). Notice that $\mathbf{A}, \mathbf{B}, \mathbf{B}_{d}, \mathbf{E}_{u}, \mathbf{E}_{d}$, are time-invariant matrices of suitable dimensions dictated by the network topology.

The system (1) is subject to hard state and input constraints describing convex polytopic sets

$$
\begin{aligned}
& \mathcal{X} \triangleq\left\{\mathbf{x}(k) \in \mathbb{R}^{n_{x}} \mid \mathbf{G x}(k) \leq \mathbf{g}\right\} \subset \mathbb{R}^{n_{x}} \quad \forall k, \\
& \mathcal{U} \triangleq\left\{\mathbf{u}(k) \in \mathbb{R}^{n_{u}} \mid \mathbf{F u}(k) \leq \mathbf{f}\right\} \subset \mathbb{R}^{n_{u}} \quad \forall k,
\end{aligned}
$$

where $\mathbf{G} \in \mathbb{R}^{c_{x} \times n_{x}}, \mathbf{g} \in \mathbb{R}^{c_{x}}, \mathbf{F} \in \mathbb{R}^{c_{u} \times n_{u}}, \mathbf{f} \in \mathbb{R}^{c_{u}}$, being $c_{x}$ and $c_{u}$ the number of state and input constraints, respectively. Moreover, for service reliability, the states are also subject to the following safety constraints:

$$
\mathbf{x}(k) \geq \mathbf{d}_{\text {net }, k} \quad \forall k,
$$

where $\mathbf{d}_{\text {net }, k} \in \mathbb{R}^{n_{x}}$ is the vector of (possibly time-varying) lower-bounds on water storages (expressed in $\mathrm{m}^{3}$ ) necessary to avoid water stock-outs.

\section{B. Model decomposition}

Once the control-oriented model is stated, it is necessary to efficiently and properly decompose the overall system into subsystems so that the DCG approach proposed in this paper can be used. The decomposition aims at achieving several benefits, such as modularity and reduction of the computational burden for each single agent, while maintaining the control performance similar to those pertaining to a centralized solution (or at most with an acceptable degradation).

The partitioning algorithm proposed in [12] automatically obtains this decomposition by identifying clusters of vertices within the graph $G(\mathcal{V}, \mathcal{E})$ that are strongly connected with each other but weakly interconnected with the other clusters. As a result, the overall DWN may be represented as a set of loosely coupled subsystems. Therefore, the system in (1) is assumed to be decomposed in $M \triangleq|\mathcal{N}|$ subsystems collected in the set $\mathcal{N}$, which are not overlapped, output decentralized and input coupled. The model for the $i$-th subsystem $S_{i}$, for $i \in\{1, \ldots, M\}$, can be written as

$$
\begin{aligned}
\mathbf{x}_{i}(k+1) & =\mathbf{A}_{i} \mathbf{x}_{i}(k)+\mathbf{B}_{s h, i} \mathbf{u}_{i}(k)+\mathbf{B}_{d, i} \mathbf{d}_{i}(k), \\
\mathbf{0} & =\mathbf{E}_{s h, i} \mathbf{u}_{i}(k)+\mathbf{E}_{d, i} \mathbf{d}_{i}(k),
\end{aligned}
$$

where $\mathbf{E}_{s h, i}$ and $\mathbf{E}_{s h, i}$ are matrices whose dimensions depend on the number of shared inputs of subsystem $S_{i}$. For simplicity, hereafter the following constraints are considered:

$$
\begin{aligned}
& \mathbf{x}_{\min , i} \leq \mathbf{x}_{i}(k) \leq \mathbf{x}_{\max , i}, \quad \forall k \\
& \mathbf{u}_{\min , i} \leq \mathbf{u}_{i}(k) \leq \mathbf{u}_{\max , i}, \quad \forall k,
\end{aligned}
$$

which correspond to a special case of the more general classes (2a) and (2b).

\section{System Operational Goals}

The main goal of the operational control of DWN at transport level is to fully satisfy the water demands while, at the same time, optimizing the management policies expressed as a multi-objective optimal control problem. As better explained in [4], common operational goals for the management of DWNs are of three types: (i) economic, providing a reliable water supply minimising water production and water transport costs; (ii) safety, guaranteeing the availability of enough water in each storage tank to satisfy its underlying stochastic demand; and (iii) smoothness, operating the DWN under smooth control actions. The economic and smoothness goals can be achieved by minimising the following performance indicators

$$
\begin{aligned}
J_{E, k}^{i} & \triangleq\left\|\left(\boldsymbol{\alpha}_{1}^{i}+\boldsymbol{\alpha}_{2, k}^{i}\right)^{\mathrm{T}} \mathbf{u}_{i}(k)\right\|_{1, \mathbf{W}_{e}^{i},} \\
J_{\Delta U, k}^{i} & \triangleq\left\|\Delta \mathbf{u}_{i}(k)\right\|_{2, \mathbf{W}_{u}^{i}}^{2},
\end{aligned}
$$

where $J_{E}^{i} \in \mathbb{R}_{\geq 0}$ represents the economic cost of network operation taking into account water production cost $\boldsymbol{\alpha}_{1} \in$ $\mathbb{R}^{n_{u}}$ and water pumping cost $\boldsymbol{\alpha}_{2} \in \mathbb{R}^{n_{u}^{i}}$, which change every time instant $k$ according to the variable electric tariff; $J_{\Delta U}^{i} \in$ $\mathbb{R}_{\geq 0}$ represents the penalisation of control signal variations $\Delta \mathbf{u}(k) \triangleq \mathbf{u}(k)-\mathbf{u}_{k-1}$, of use to extend actuators life and assure a smooth operation; $\|\cdot\|_{p, \mathbf{W}}$ is a weighted $p$-norm; and $\mathbf{W}_{e}^{i}, \mathbf{W}_{u}^{i}$ are diagonal matrices that weight each decision variable in the corresponding control objective.

The second goal is enforced by the safety constraint (3), which can be conveniently reformulated as the soft constraint

$$
\mathbf{x}_{i}(k) \geq \mathbf{x}_{s, k}^{i}-\boldsymbol{\xi}_{i}(k) \geq \mathbf{0} \quad \forall k,
$$


where $\mathbf{x}_{\mathrm{s}}^{i} \in \mathbb{R}^{n_{x}^{i}}$ is a vector of suitably determined safetyvolume thresholds in $\mathrm{m}^{3}$, and $\boldsymbol{\xi} \in \mathbb{R}^{n_{x}}$ represents the amount of volume going down from the desired safety thresholds. As a result, the performance index

$$
J_{S, k}^{i} \triangleq\left\|\boldsymbol{\xi}_{i}(k)\right\|_{2, \mathbf{W}_{x}^{i}}^{2}
$$

is consequently stated.

\section{DCG STRATEGY APPLIED TO DWN OPERATIONAL CONTROL}

The DCG scheme of interest here is depicted in Fig. 1. There, a set $\mathcal{N}$ of $M$ stable plants are supervised by local CG (agents) devices via command sequences $\mathbf{u}_{i}(k)$. In this case, it is convenient to recast the discrete DAEs in (4) as follows:

$$
\begin{aligned}
\mathbf{x}_{i}(k+1) & =\mathbf{A}_{i} \mathbf{x}_{i}(k)+\mathbf{B}_{i} \mathbf{u}_{i}(k)+\sum_{j \in \mathcal{N}_{i}} \mathbf{B}_{j} \mathbf{u}_{j}(k)+\mathbf{B}_{d,} \mathbf{d}_{i}(k)(9 \mathrm{a}) \\
\mathbf{0} & =\mathbf{E}_{u, i} \mathbf{u}_{i}(k)+\sum_{j \in \mathcal{N}_{i}} \mathbf{E}_{u, j, i} \mathbf{u}_{j}(k)+\mathbf{E}_{d, i} \mathbf{d}_{i}(k), \quad \text { (9b) }
\end{aligned}
$$

where the flow $\mathbf{u}_{i}(k)$ is supervised by the $i$-th agent while all the other flows $\mathbf{u}_{j}(k)$ by a corresponding $j$-th agent each, all belonging to the set $\mathcal{N}_{i}$ of neighboring agents for the $i$-th agent.

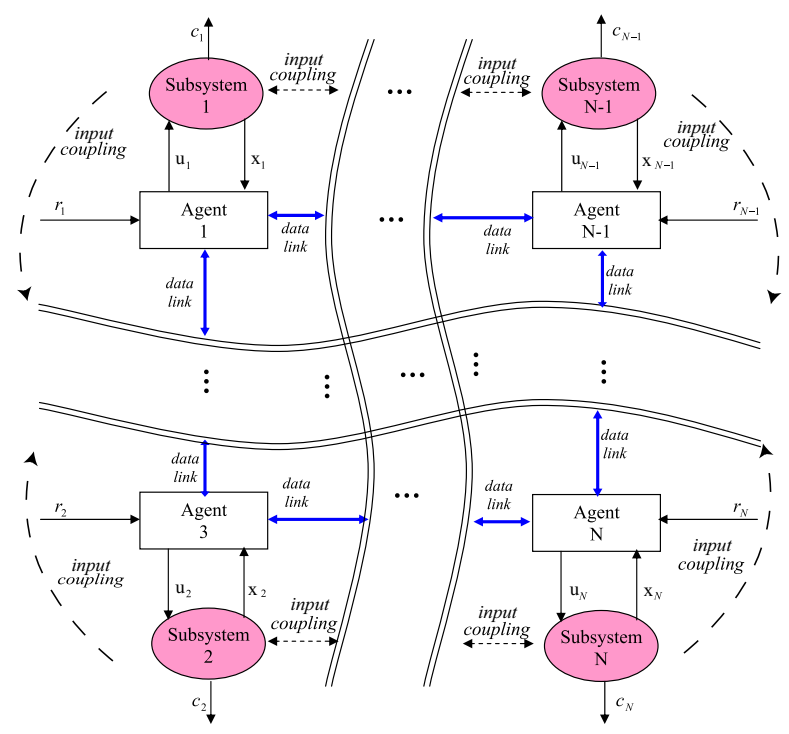

Fig. 1. DCG Scheme

More formally, the local task to be fulfilled by each agent at each time $k$ is that of locally computing, on the basis of the desired reference $\mathbf{r}_{i}(k)$, measured state $\mathbf{x}_{i}(k)$ and external input signals $\mathbf{u}_{j}(k)$ and $d_{i}(k)$, the command $\mathbf{u}_{i}(k)$ as the best approximation of $\mathbf{r}_{i}(k)$ under the following pointwisein-time constraints:

$$
\mathbf{c}_{i}(k) \in \mathcal{C}_{i},
$$

to be hold true along the system trajectories generated by the CG. In particular,

$$
\mathbf{c}_{i}(k):=\mathbf{C}_{i} \mathbf{x}_{i}(k)+\mathbf{L}_{i} \mathbf{u}_{i}(k),
$$

where $\mathcal{C}_{i}$ is a convex set and $\mathbf{C}_{i}:=\left[\mathbf{I}_{n_{x}^{i}}^{T}, \mathbf{0}_{n_{u}^{i}}^{T}\right]^{T}$ and $\mathbf{L}_{i}:=$ $\left[\mathbf{0}_{n_{x}^{i}}^{T}, \mathbf{I}_{n_{u}^{i}}^{T},\right]^{T}$. For the problem at hand, the set $\mathcal{C}_{i}$ is defined

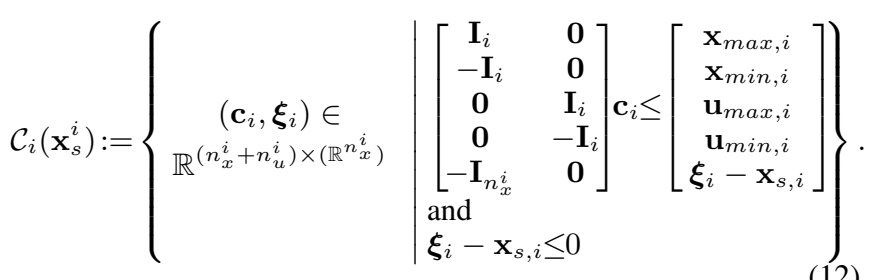

The above task is accomplished through the non-iterative optimization procedure presented in [16], where the idea is that agents that are not jointly involved in any coupling constraint can simultaneously update their control actions without violating constraints. To this aim, agents are grouped into particular subsets (or turns).

Definition 1: (Turn) A turn $\mathcal{T} \subset \mathcal{N}$ is a subset of nonneighboring nodes, i.e., $\forall i, j \in \mathcal{T}$ such that $i \neq j, j \notin \mathcal{N}_{i}$ (none of them is a neighbor of the others).

In the previous work [16], the above agent partitions were exploited to build up a distributed strategy where at each sampling time, on the basis of a round-robin policy for the turns, only agents belonging to a turn are allowed to update simultaneously their commands $\mathbf{u}_{i}(k)$, while all agents in all other turns keep applying constantly their current commands until their turn becomes active. Here, by following the procedure sketched in [15], a faster approach is proposed, where the above round-robin process among turns is completed within a sampling time. The approach clearly requires agents to know when to optimize their local commands $\mathbf{u}_{i}$. To this end, assume that

- a communication network linking neighboring agents exists and it is modeled as a graph $\Gamma$;

- a sequence of turns $\mathcal{T}_{1}, \mathcal{T}_{2}, \ldots \mathcal{T}_{q}$ which covers completely $\Gamma$ has been determined.

Roughly speaking, at each time $k$, for each turn, agents carry out the following basic actions:

1) receive finite sequences $\mathbf{u}_{j}(l \mid k), l=0,1, \ldots$ of computed flows from previous updating neighboring agents and $\mathbf{u}_{j}(l \mid k-1), l=0,1, \ldots$ from next updating neighboring agents;

2) compute $\mathbf{u}_{i}(k), l=0,1, \ldots$ according to the minimization of a proper optimization program;

3) transmit $\mathbf{u}_{i}(l \mid k), l=0,1, \ldots$ to neighboring agents;

4) apply $\mathbf{u}_{i}(0 \mid k)$.

The local optimization problem solved by each agent aims at selecting, at each time $k$, an open-loop virtual command sequence $\mathbf{u}_{i}(l \mid k) \equiv \mathbf{w}_{i}$, chosen in such a way that, if constantly applied to the system over a semi-infinite horizon $l \in[0, \infty)$, from the initial state $\mathbf{x}_{i}(k)$, it would never produce constraint violations. Moreover, in order to take into account the algebraic-equations (1b), the following set is introduced:

$$
\begin{gathered}
\mathcal{W}_{i}\left(\left\{\mathbf{u}_{j}\right\}_{j \in \mathcal{N}_{i}}, \mathbf{d}_{i}\right)=\left\{\mathbf{w} \in \mathbb{R}^{n_{u}^{i}}: \mathbf{E}_{u, i} \mathbf{w}_{i}\right. \\
\left.+\sum_{j \in \mathcal{N}_{i}} \mathbf{E}_{u, j, i} \mathbf{u}_{j}(k)+\mathbf{E}_{d, i} \mathbf{d}_{i}=0\right\}
\end{gathered}
$$


The applied command $\mathbf{w}_{i}$ is chosen in such a way that the future predictions (virtual evolutions) of the $\mathbf{c}_{i}$-variable along the virtual time $l$ under a constant virtual command $\mathbf{u}_{i}(l \mid k) \equiv$ $\mathbf{w}_{i}$ from the initial state $\mathbf{x}_{i}$ (at virtual time $l=0$ )

$$
\begin{aligned}
& \mathbf{c}_{i}\left(l, \mathbf{x}_{i}, \mathbf{w}_{i},\left\{\mathbf{u}_{j}\right\}_{j \in \mathcal{N}_{i}}, \mathbf{d}_{i}\right)= \\
& \mathbf{C}_{i}\left(\mathbf{A}_{i}^{l} \mathbf{x}_{i}+\sum_{j=0}^{l-1} \mathbf{A}_{i}^{l-j-1}\left(\mathbf{B}_{i} \mathbf{w}_{i}+\sum_{j \in \mathcal{N}_{i}} \mathbf{B}_{j, i} \mathbf{u}_{j}+\mathbf{B}_{d, i} \mathbf{d}_{i}\right)\right)+\mathbf{L}_{i} \mathbf{w}_{i}
\end{aligned}
$$

do not violate constraints $\mathbf{c}_{i}\left(l, \mathbf{x}_{i}, \mathbf{w}_{i},\left\{u_{j}\right\}_{j \in \mathcal{N}_{i}}, \mathbf{d}_{i}\right) \in$ $\mathcal{C}_{i}, \forall l \in \mathbb{Z}_{+}$.

In the case of a constant disturbance $\mathbf{d}_{i}(l \mid k)=\mathbf{d}_{i}$, this is obtained by selecting the applied command as follows:

$\mathbf{w}_{i} \in \mathcal{V}_{i}\left(\mathbf{x}_{i},\left\{\mathbf{u}_{j}\right\}_{j \in \mathcal{N}_{i}}, \mathbf{d}_{i}\right):=\left\{\mathbf{w}_{i} \in \mathcal{W}(\mathbf{d}): \exists \boldsymbol{\xi}_{i}(l)\right.$

such that $\left.\left.\mathbf{c}\left(l, \mathbf{x}_{i}, \mathbf{w}_{i},\left\{u_{j}\right\}_{j \in \mathcal{N}_{i}}, \mathbf{d}_{i}\right), \boldsymbol{\xi}_{i}(l)\right) \in \mathcal{C}\left(\mathbf{x}_{s}\right), \forall l \in \mathbb{Z}_{+}\right\}$.

It is worth mentioning that, if $\mathbf{A}_{i}$ is Schur, the set $\mathcal{V}\left(\mathcal{W}_{i}\left(\left\{\mathbf{u}_{j}\right\}_{j \in \mathcal{N}_{i}}, \mathbf{d}_{i}\right)\right), \forall \mathbf{x}_{i} \in \mathbb{R}^{n_{x}^{i}}$, is convex and finitely determined, viz. there exists an a-priori known integer $l_{0}$ (see [18]) such that if $\left.\mathbf{c}\left(l, \mathbf{x}_{i}, \mathbf{w}_{i},\left\{u_{j}\right\}_{j \in \mathcal{N}_{i}}, \mathbf{d}_{i}\right), \boldsymbol{\xi}_{i}(l)\right) \in$ $\mathcal{C}_{i}\left(\mathbf{x}_{s, i}\right), l \in\left\{0,1, \ldots l_{0}\right\}$, then $\mathbf{c}\left(l, \mathbf{x}_{i}, \mathbf{w}_{i},\left\{u_{j}\right\}_{j \in \mathcal{N}_{i}}, \mathbf{d}_{i}\right) \in$ $\mathcal{C}_{i}\left(\mathbf{x}_{s, i}\right), \forall l \in \mathbb{Z}_{+}$.

Finally, the local $\mathrm{CG}$ problem is solved by choosing at each time instant $k$ a command $\mathbf{u}_{i}^{*}(k)$, which is the solution of the following convex optimization problem:

$$
\mathbf{u}_{i}^{*}(k)=\arg \min _{\mathbf{w}_{i} \in \mathcal{V}_{i}\left(\mathbf{x}_{i}(k),\left\{\mathbf{u}_{j}(k)\right\}_{j \in \mathcal{N}_{i}}, \mathbf{d}_{i}\right)} J^{*},
$$

where $J^{*}$ is computed as in (6) with

$$
J_{E, i, k} \triangleq\left\|\left(\boldsymbol{\alpha}_{1, i}+\boldsymbol{\alpha}_{2, i, k}\right)^{\mathrm{T}}\left(\mathbf{w}_{i}-\mathbf{r}_{i}(k)\right)\right\|_{1, \mathbf{w}_{e, i}} .
$$

In the case of time-varying disturbance predictions, commands $\mathbf{u}_{i}(l \mid k)$ need to be selected in a set that depends on the entire disturbance sequence $\underset{\mathbf{d}_{i}}{\rightarrow}(k) \triangleq\left[\mathbf{d}_{i}(0 \mid k), \ldots, \mathbf{d}_{i}\left(l_{0} \mid k\right)\right]$ and has the following form:

$$
\begin{aligned}
& \mathcal{V}_{i}\left(\mathbf{x}_{i}, \underline{\mathbf{d}}_{i}(k)\right) \triangleq\left\{\underset{\mathbf{w}_{i}}{\rightarrow}(l):\left\{\mathbf{w}_{i}(l) \in \mathcal{W}_{i}\left(\mathbf{d}_{i}(l)\right)\right\} \text { s.t. } \exists \boldsymbol{\xi}_{i}(l),\right. \\
& \left(\mathbf{c}\left(l, \mathbf{x}_{i}, \mathbf{w}_{i}(l),\left\{u_{j}(l)\right\}_{j \in \mathcal{N}_{i}}, \mathbf{d}_{i}(l), \boldsymbol{\xi}_{i}(l)\right) \in \mathcal{C}\left(\mathbf{x}_{s, i}\right),\right. \\
& \left.\forall l \in\left\{0,1, \ldots, l_{0}\right\}\right\}
\end{aligned}
$$

The procedure previously explained and discussed is summarized in Algorithm 1.

\section{ApPliCATION TO THE BARCELONA DWN}

\section{A. Case Study Description}

The selected case study is an aggregate and representative version of the Barcelona DWN. In this model, some network water demand sectors are concentrated in a single demand. Similarly, some tanks are aggregated in a single element and the corresponding actuators are considered as a single pumping station or valves [19]. The model consists of 17 tanks, 61 actuators, 25 measured demands and 11 nodes (see Figure 2). Water is taken from rivers Besòs and Ter, particularly from three sources named Abrera, Llobregat and Cardedeu where there are, respectively, three water treatment plants.

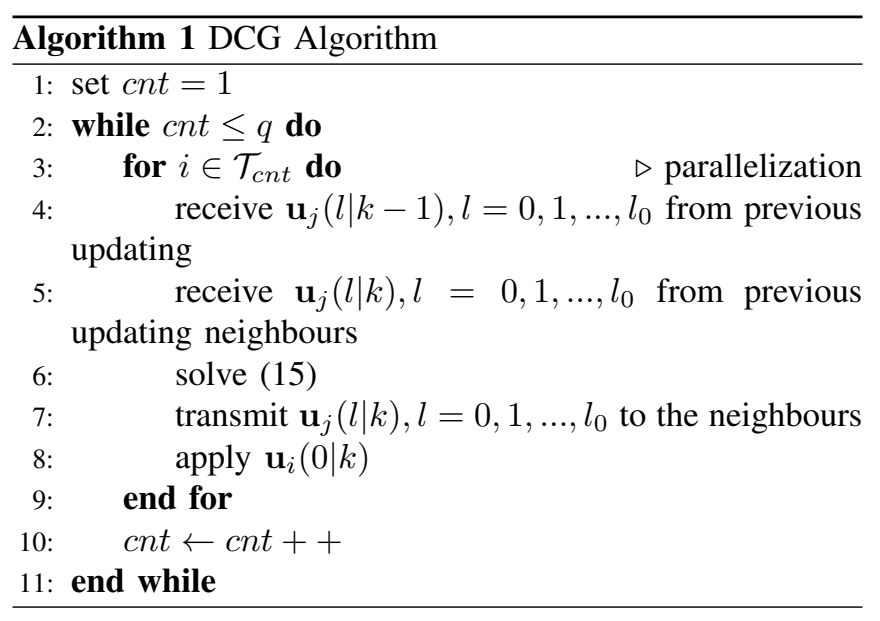

\section{B. Case Study Partitioning}

Using the partitioning algorithm presented in [12], the aggregate model of the Barcelona DWN is decomposed into three subsystems, as depicted in Fig. 2 with different colours. DWN subsystems are composed by the following elements:

- Subsystem 1 (red): Composed by tanks $x_{i}, i \in\{1,2\}$, inputs $u_{j}, j \in\{1: 5\}$, demands $d_{l}, l \in\{1,2,3\}$, and nodes $n_{q}, q \in\{1,2\}$.

- Subsystem 2 (green): Composed by tanks $x_{i}, i \in$ $\{3,4,5,12,17\}$, inputs $u_{j}, j \in\{7: 16,18,19,25,26$, $32,34,40,41,47,48,56,60\}, \quad$ demands $d_{l}$, $l \in\{4: 7,15,18,22\}$, and nodes $n_{q}, q \in\{3,4,7\}$.

- Subsystem 3 (blue): Composed by tanks $x_{i}, i \in\{6$ : $11,13: 16\}$, the inputs $u_{j}, j \in\{6,17,20: 24,27$ : $31,33,35: 39,42: 46,49: 55,57,58,59,61\}$, demands $d_{l}, l \in\{8: 14,16,17,19,20,21$, $23,24,25\}$, and nodes $n_{q}, q \in\{5,6,8: 11\}$.

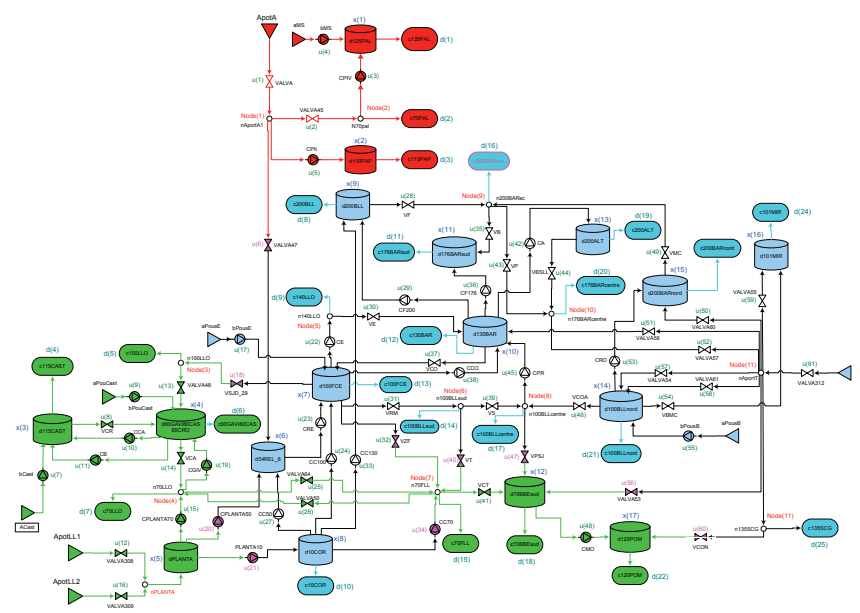

Fig. 2. Case Study: Aggregate model of the Barcelona DWN. Subsystems are highlighted by different colors: red (Subsystem 1), green (Subsystem 2), blue (Subsystem 3)

\section{Closed-loop Setup}

All results have been obtained by considering a four-day real-demand scenarios (with 1 hour of sampling time), and $H_{p}=H_{u}=i_{0}=24$. Control objectives in (6) are prioritised 
with the following weights: $W_{e}=100, W_{x}=10$ and $W_{u}=1$, following a trial-and-error tuning strategy. The network has been simulated by using the same model used to design the controller but fed with real water demands. The network model has been calibrated and validated by using real data provided by $\mathrm{AGBAR}^{1}$. Concerning the DCG, it is worth to mention that agents are grouped into two turns $\mathcal{T}_{1}=\{$ Agent 3 (blue) $\}, \mathcal{T}_{2}=\{$ Agent 1 (red), Agent 2 (green) $\}$. All simulations have been undertaken by using the Yalmip interpreter [20] and the CPLEX solver, all under MATLAB $^{\complement} 8.2$ environment, running on an Intel ${ }^{\complement}$ Core i5-3330 machine with $3.3 \mathrm{GHz}$ and $8 \mathrm{~GB}$ RAM.

\section{Results, Comparison and Discussion}

In order to show the effectiveness of the DCG approach proposed in this paper, it will be compared against the decentralized MPC (DMPC) and multi-layer decentralized MPC (ML-DMPC) approaches proposed in [12] and [17], respectively, which were also applied to the control of DWNs using the same case study of Barcelona.

Table I shows the results achieved by the DCG approach as well as those obtained when the ML-DMPC and DMPC approaches and their centralized versions are used ${ }^{2}$. From these results it can be seen that the DCG approach achieves a performance that is quite close to that pertaining to the centralized CG scheme. This means that the DCG implementation results to be quite effective.

On the other hand, the DCG approach clearly achieves better performance when compared with the DMPC approach. This is because the DMPC approach has only a local view of the control problem, which tends to overemphasize the local objectives (electrical costs). On the contrary, when the ML-DMPC is used, which takes into account also the global objectives (water costs), the results are closer to that of the DCG approach.

Figures 3 and 4 show comparatively the evolutions of a tank volume and a valve flow when the DCG and DMPC approaches are used. On the other hand, Figure 5 shows the different usage of the available water sources done by each approach. Also notice the cyan areas in all figures that delimit time intervals where the cost of electricity is the lowest with respect to the remainder of the day. As a matter of fact, it results that all approaches try to move water into the tank during the night when the energy is cheaper as it can be seen in Figure 3.

Interestingly enough, these figures show different management behaviors produced by the different approaches, also confirmed by the results reported in Table I. Specifically, the total cost achieved by DCG and ML-DMPC are quite similar and better than that achieved by DMPC. This can be seen for example in the management of valve $u_{54}$ in Figure 4 and in Table I. However, unlike DMPC and ML-DMPC, it can be seen from Figure 3 that DCG makes a stronger use of

\footnotetext{
${ }^{1}$ Aigües de Barcelona, S.A., the company that manages the Barcelona DWN.

${ }^{2}$ Notice that the economic costs in Table I are given in economic units rather than real values (Euro) due to confidentiality reasons.
}

pumps in order to obtain a better water management (see Figure 5). Such a behavior translates into lower water costs at the price of increased electric costs while complementary behaviors are observed for DMPC and ML-DMPC.

Finally, centralized MPC and CG strategies yield quite similar results and management policies. Minor differences can been observed, mainly related to the statement of the optimization problems for each strategy. In particular, CG achieves a slightly better performance than the MPC strategy in terms of electric cost that nevertheless weakly outperforms CG in terms of water cost. Regarding the total cost, MPC yields in lower values mainly towards the final of the simulation. Finally, the computational burden is practically the same with a slight advantage for the CG setup. The centralized methods present, as expected, a quite higher CPU time per step with respect to the decentralized approaches. Notice also that, despite all simulations have considered a four-day scenario, the closed-loop system reached its steady state at the end of the second day of simulation.

TABLE I

COMPLETE BREAK-DOWN OF ECONOMIC COSTS FOR THE DIFFERENT APPROACHES

\begin{tabular}{lccccc}
\hline & & DAY 1 & DAY 2 & DAY 3 & DAY 4 \\
\hline \multirow{4}{*}{ WATER CosT } & DMPC & 35.79 & 42.65 & 42.10 & 41.51 \\
& DCG & 14.76 & 25.72 & 27.13 & 26.66 \\
& MPC & 14.46 & 25.87 & 27.55 & 27.63 \\
& CG & 14.49 & 26.08 & 27.87 & 27.94 \\
\hline & DMPC & 10.93 & 12.32 & 12.06 & 11.78 \\
ELECTRIC COST & DCG & 23.88 & 23.65 & 23.98 & 23.99 \\
& ML-DMPC & 12.80 & 13.45 & 13.27 & 13.03 \\
& MPC & 21.52 & 21.68 & 21.87 & 21.87 \\
& CG & 21.44 & 21.60 & 21.79 & 21.79 \\
\hline \multirow{5}{*}{ TOTAL CosT } & DMPC & 46.71 & 54.97 & 54.16 & 53.29 \\
& DCG & 38.64 & 49.37 & 51.11 & 50.65 \\
& ML-DMPC & 39.99 & 47.46 & 47.46 & 45.80 \\
& MPC & 35.99 & 47.55 & 49.42 & 49.50 \\
& CG & 35.93 & 47.68 & 49.65 & 49.73 \\
\hline \multirow{5}{*}{ CPU TIME } & DMPC & 7.78 & 8.43 & 8.51 & 8.59 \\
& DCG & 7.75 & 8.39 & 8.49 & 8.54 \\
& ML-DMPC & 7.82 & 8.46 & 8.79 & 8.64 \\
& MPC & 9.48 & 10.31 & 10.71 & 10.65 \\
& CG & 9.34 & 10.11 & 10.23 & 10.29 \\
\hline \hline
\end{tabular}

\section{Conclusions}

This paper has proposed the application of a DCG strategy for the operational control of DWNs. The application of the $\mathrm{CG}$ approach to the problem at hand required a slightly modified of the theory in order to face with marginal stable systems, incorporating equality constraints and dealing with disturbance rejection goals. The provided simulation results obtained by using the Barcelona DWN as a case study and the comparison with two DMPC schemes have shown similarities with some noticeable difference in managing the system. In particular, all approaches shown similar computational burdens and total management costs although the CG approach seems to be computationally less demanding. However, the water and electricity costs are minimized differently by the various schemes, giving rise to different management strategies, although their sum (the total cost) resulted quite the same for all the contrasted strategies. 


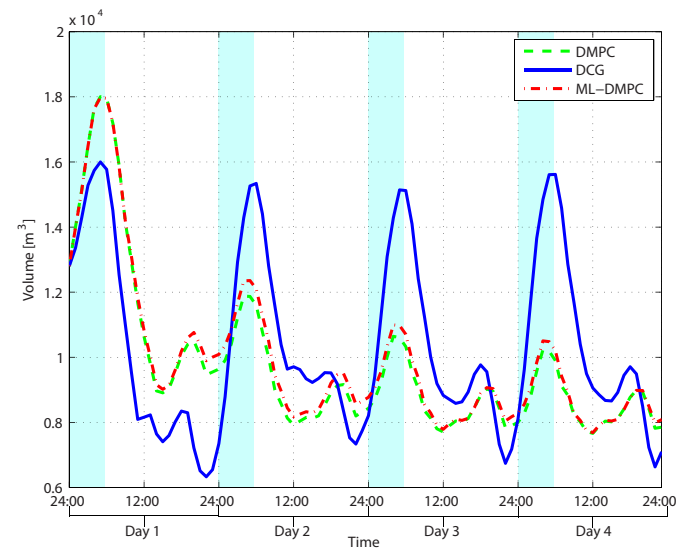

Fig. 3. Resultant volume related to tank $x(10)$ (d130BAR)

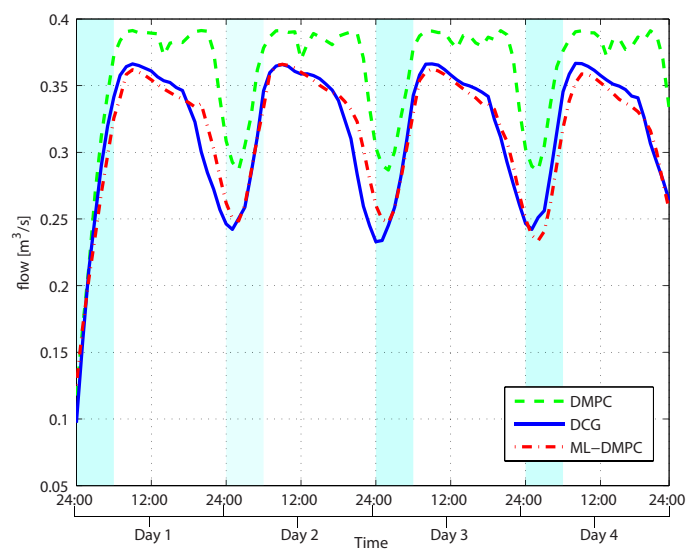

Fig. 4. Computed flow related to valve $u(54)$.

\section{REFERENCES}

[1] A. Castelletti, F. Pianosi, and R. Soncini-Sessa, "Stochastic and robust control of water resource systems: Concepts, methods and applications," in System Identification, Environmental Modelling, and Control System Design. Springer London, 2012, pp. 383-401.

[2] G. Cembrano, J. Quevedo, V. Puig, R. Perez, J. Figueras, J. M. Verdejo, I. Escaler, G. Ramon, G. Barnet, P. Rodriguez, and M. Casas, "PLIO: a generic tool for real-time operational predictive optimal control of water networks," Water Science \& Technology, vol. 64, no. 2, pp. 448459, 2011.

[3] A. El Mouatasim, R. Ellaia, and A. Al-Hossain, "A continuous approach to combinatorial optimization: application of water system pump operations," Optimization Letters, vol. 6, pp. 177-198, 2012.

[4] C. Ocampo-Martinez, V. Puig, G. Cembrano, and J. Quevedo, "Application of predictive control strategies to the management of complex networks in the urban water cycle," Control Systems, IEEE, vol. 33, no. 1, pp. 15-41, 2013.

[5] I. Pulido-Calvo, J. Gutiérrez-Estrada, and D. Savic, "Heuristic modelling of the water resources management in the Guadalquivir river basin, southern Spain," Water Resources Management, vol. 26, pp. 185-209, 2012.

[6] J. Vieira, M. Cunha, L. Nunes, J. Monteiro, L. Ribeiro, T. Stigter, J. Nascimento, and H. Lucas, "Optimization of the operation of largescale multisource water-supply systems," Journal of Water Resources Planning and Management, vol. 137, no. 2, pp. 150-161, 2011.

[7] J. Grosso, C. Ocampo-Martinez, and V. Puig, "Learning-based tuning of supervisory model predictive control for drinking water networks," Engineering Applications of Artificial Intelligence, vol. 26, no. 7, pp. $1741-1750,2013$.
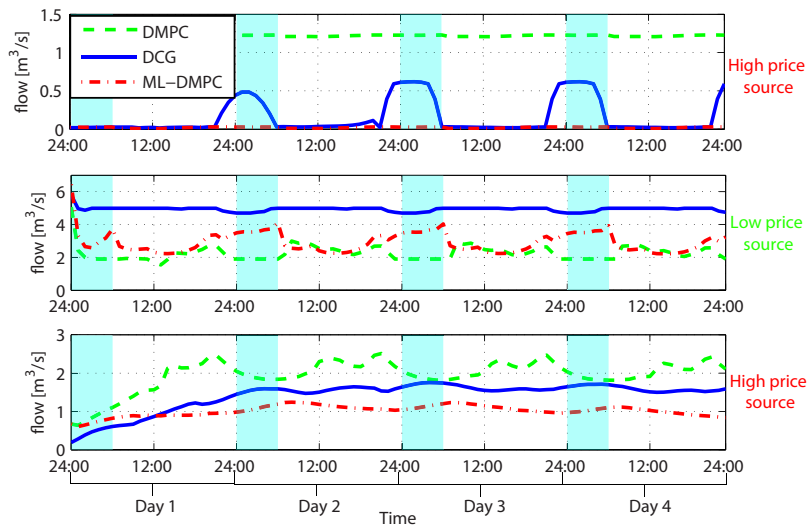

Fig. 5. Comparison of the network inflow from the main water sources: (top) Abrera $(u(1))$,(middle) Llobregat $(u(12)+u(16))$, and (bottom) Cardedeu $(u(61))$.

[8] J. Maciejowski, Predictive control with constraints. Essex, England: Prentice Hall, 2002.

[9] D. Angeli and E. Mosca, "Command governor for nonlinear systems under constraints," IEEE Trans. on Automatic Control, vol. 44, pp. 816-820, 1999.

[10] F. Tedesco, C. Ocampo-Martinez, A. Casavola, and V. Puig, "On the comparison of predictive control and command governor approaches for operational control of drinking water networks: A case study," in Proceedings of 19th IFAC World Congress, Cape Town, South Africa, August 24 - 292014.

[11] J. Maestre and R. E. Negenborn, Distributed Model Predictive Control Made Easy, ser. Intelligent Systems, Control and Automation: Science and Engineering. Springer, 2014.

[12] C. Ocampo-Martinez, S. Bovo, and V. Puig, "Partitioning approach oriented to the decentralised predictive control of large-scale systems," Journal of Process Control, vol. 21, no. 5, pp. 775 - 786, 2011.

[13] F. Tedesco, A. Casavola, and E. Garone, "Distributed command governor strategies for constrained coordination of multi-agent networked systems." in 2012 American Control Conference (ACC), June 27 - 29 2012, pp. 6005-6010.

[14] F. Tedesco, A. Casavola, and E. Garone, "A distributed parallel command governor strategy for the coordination of multi-agent networked systems," in 4th IFAC Nonlinear Model Predictive Control Conference, vol. 4, August 23 - 27, 2012, pp. 478-483.

[15] A. Casavola, E. Garone, and F. Tedesco, "A distributed multi-agent command governor strategy for the coordination of networked interconnected systems," IEEE Transactions on Automatic Control, vol. 59, no. 8, p. 2099 âĂŞ2112, 2014.

[16] A. Casavola, E. Garone, and F. Tedesco, "Scalability and performance improvement of distributed sequential command governor strategies via graph colorability theory," in Proceedings of 19th IFAC World Congress, Cape Town, South Africa, August 24 - 29, 2014.

[17] C. Ocampo-Martinez, V. Puig, J. Grosso, and S. Montes-de Oca, DMPC made easy. Springer, 2014, ch. Multi-layer Decentralized Model Predictive Control of Large-Scale Networked Systems, pp. 495 -515 .

[18] E. Gilbert, I. Kolmanovsky, and K. Tan, "Command governor for nonlinear systems under constraints," International Journal on Robust and Nonlinear Control, vol. 5, pp. 487-504, 1995.

[19] C. Ocampo-Martinez, V. Puig, G. Cembrano, R. Creus, and M. Minoves, "Improving water management efficiency by using optimization-based control strategies: the Barcelona case study," Water Science \& Technology: Water supply, vol. 9, no. 5, pp. 565-575, 2009.

[20] J. Löfberg, "Yalmip : A toolbox for modeling and optimization in MATLAB," in Proceedings of the CACSD Conference, Taipei, Taiwan, 2004. [Online]. Available: http://users.isy.liu.se/johanl/yalmip

[21] D. Šiljak, Decentralized control of complex systems. Academic Press, 1991. 